\title{
Probing the erosion and cohesion of a granular raft in motion
}

\author{
Antoine Lagarde $\odot$ and Suzie Protière* \\ Institut Jean Le Rond D 'Alembert, CNRS, Sorbonne Université, UMR 7190, F-75005 Paris, France
}

\begin{abstract}
Particles at a liquid interface can interact through capillarity, and depending on their size, shape, or surface chemistry, it may lead to their aggregation. Yet the two-dimensional structures thus created can be eroded by strong enough flows or as described in this paper via their own motion along a deformed interface. Here we experimentally highlight an unexpectedly high resistance of such a granular raft to erosion, which cannot be explained by the usual linear calculation of the capillary interaction. To clarify this result, we develop a model experiment in which two particles are horizontally moved along the liquid interface at a chosen velocity until they separate. We deduce again a cohesive force that exceeds the capillary attraction expected by one or two orders of magnitude. A precise description of the geometry of the contact line around a sphere and of its vertical position
\end{abstract} seems to account rather well for the experimental results.

I. INTRODUCTIONParticles placed in a Newtonian fluid may undergo attractive interactions and form aggregates. These aggregates under flow can break up or change shape [1,2]. This phenomenon is observed in many consumer products (cosmetics, food, and paint, for example). Since the work of Camoin et al. [3], some studies have focused on the behavior of twodimensional (2D) aggregates stuck at a liquid interface to understand their erosion and break-up. Indeed, in this case, reconfigurations are effortlessly observable as the object remains on the same plane throughout an experiment, and the hydrodynamic forces at play may then be investigated easily. With different external stresses, a monolayer of particles can undergo several types of structural changes, from a simple reorganization of its internal structure for low stresses $[4,5]$ to the formation of cracks or folds [6,7] up to its break-up for strong enough external flows. Two break-up mechanisms have been identified: erosion, where the aggregate loses single particles, and fragmentation, during which the 2D structure breaks into several large pieces. Numerical simulations [8] along with experiments $[9,10]$ have tried to characterize the relative importance of both phenomena. Nevertheless, the mechanisms responsible for the resistance of an aggregate to erosion are still far from being understood.

In this paper, we focus on the erosion of a 2D dense aggregate at an oil-water interface, from an experimental perspective. The granular raft, formed by the aggregation of dense spherical beads into an axisymmetric object [11,12], moves along a curved interface in a quiescent liquid. As a consequence, unlike most previous studies, the drag experienced results from its own motion instead of an externally imposed flow. From the observation of its erosion, we measure the critical force needed to extract a particle from its edge and deduce a lower boundary for the cohesive force of a raft. We then develop a model experiment to investigate the force of cohesion between two beads in contact at a liquid interface. A quantitatively good agreement is found between our data and a theoretical description of the geometry of the contact line around a sphere. Finally, based on the 

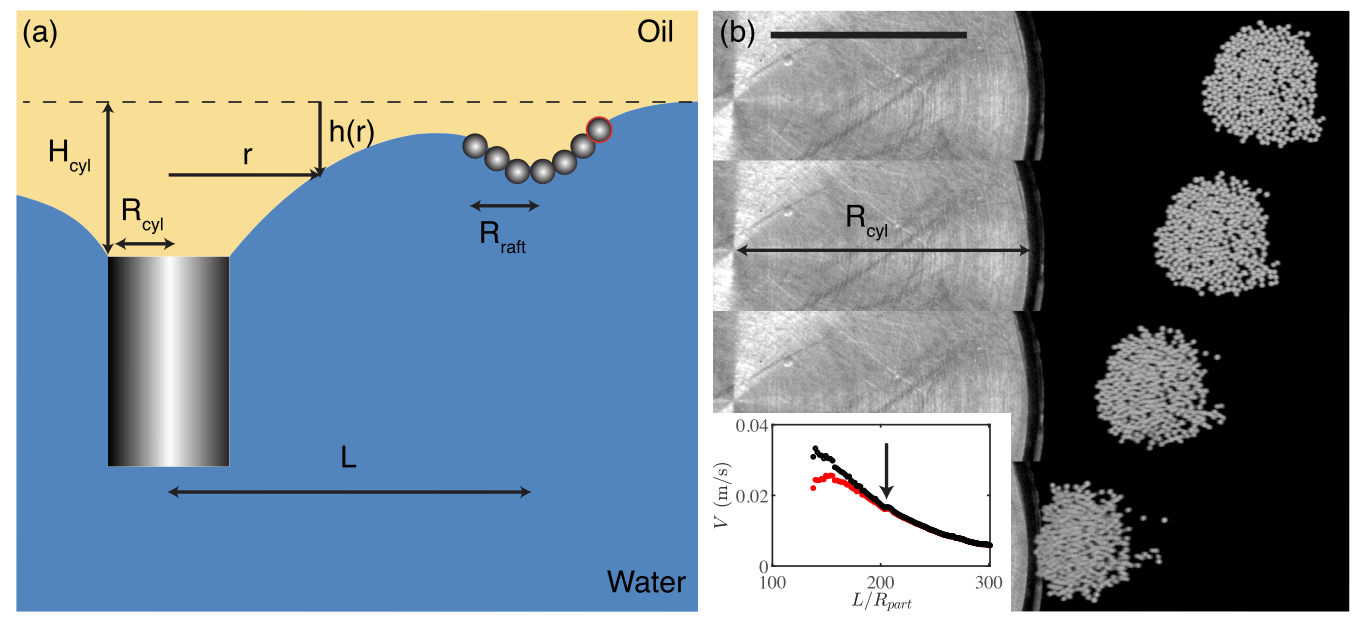

FIG. 1. Erosion of a raft: experimental setup and visualization of a typical experiment. (a) Schematic representation of the experimental configuration. A cylinder of radius $R_{\text {cyl }}$ deflects downwards an oil-water interface at a depth $H_{\text {cyl }}$, the interface being pinned at the perimeter of the cylinder. A granular raft of radius $R_{\text {raft }}$ is formed far away from the cylinder and is attracted by the cylinder through the deflection of the interface. (b) Time lapse of a granular raft experiencing erosion during its motion along a deformed oil-water interface. The raft is made of approximately 375 ceramic beads $\left(\rho_{s}=3800 \mathrm{~kg} \mathrm{~m}^{-3}, R_{\text {part }}=0.125 \mathrm{~mm}\right)$, and is moving towards a cylinder of radius $R_{\text {cyl }}=15 \mathrm{~mm}$ at a depth $H_{\text {cyl }}=10 \mathrm{~mm}$. The movie is taken from above. Time between two images: $0.15 \mathrm{~s}$. Scale bar: $10 \mathrm{~mm}$ (see Supplemental Material Movie 1 [16]). Inset: Speed of the granular raft, as a function of its distance from the center of the cylinder $L$, nondimensionalized by the radius of a particle. Red curve: horizontal component of the speed. Black curve: Total speed of the raft. The arrow indicates the moment of the first detachment of a particle from the raft.

two-bead experiment, we bring some insight into the mechanisms responsible for a granular raft's resistance to erosion.

\section{EROSION OF A GRANULAR RAFT}

In a typical experiment, as represented in Fig. 1(a), a circular metal cylinder (radius $R_{\text {cyl }}=15$ $\mathrm{mm}$ ) is placed at an oil-water interface (kinematic viscosity of the silicone oil $v=50 \times 10^{-3} \mathrm{~m}^{2} \mathrm{~s}$ ${ }^{-1}$, density $\rho_{o}=960 \mathrm{~kg} \mathrm{~m}^{-3}$, oil-water surface tension $\gamma=38 \mathrm{mN} \mathrm{m}^{-1}$ ). Elsewhere in the water tank, particles are sprinkled from above and start to aggregate once they reach the oil water interface due to capillarity [13]. They thus form a large axisymmetric monolayer of particles. Two types of ceramic beads are used in the experiment, with different densities and sizes $\left(\left\{\rho_{\text {part }}\right.\right.$; $\left.R_{\text {part }}\right\}=\left\{3800 \mathrm{~kg} \mathrm{~m}^{-3} ; 0.125 \mathrm{~mm}\right\}$ or $\left.\left\{6000 \mathrm{~kg} \mathrm{~m}^{-3} ; 0.1 \mathrm{~mm}\right\}\right)$. Their high-density results in a strong gravitational interaction. Once the granular raft is formed, the cylinder is moved downwards until the desired depth $H_{\mathrm{cyl}}$, and because the curvature of the interface acts as an external field $[14,15]$, the granular raft naturally follows the slope until it reaches the cylinder. The experiment is recorded from above at 100 frames per second, for different numbers $n_{\text {raft }}$ of particles and different cylinder depths. For low vertical deflections, the granular raft moves towards the cylinder without any internal motion of its constitutive elements. As $H_{\text {cyl }}$ is increased, slight rearrangements of the particles inside the raft may be observed, leading in some cases to erosion, as illustrated in Fig. 1(b)(see also Supplemental Material Movie 1 [16]). Because this erosion process depends not only on the parameters we can control, but also on the specific geometrical arrangement of the particles inside the raft during the aggregation process, the same experiment is reproduced several times for each pair of parameters $\left(R_{\text {raft }}, H_{\text {cyl }}\right)$. 
We can now investigate the erosion process and describe the force exerted by a raft on its constitutive element. From the top view, we have access only to the horizontal component of the speed. However, the vertical projection is accessible through the knowledge of the vertical position of the interface, which is obtained by an equilibrium between the hydrostatic pressure and the pressure jump due to the curvature, leading to the following system of equations (for cylindrical coordinates centered in the middle of the cylinder):

$$
\begin{aligned}
& \gamma\left(\frac{h^{\prime \prime}}{1+h^{\prime 2}}+\frac{h^{\prime}}{r}\right)-\left(\rho_{w}-\rho_{o}\right) g h \sqrt{1+h^{\prime 2}}=0 \\
& h(r \rightarrow \infty)=0 \\
& h\left(r=R_{\text {cyl }}\right)=H_{\text {cyl }}
\end{aligned}
$$

with $r$ the radial coordinate, $h$ the height of the interface ( $h=0$ for a flat interface), $h^{\prime}$ the derivative of $h$ with respect to $r$, and $\rho_{w}$ the density of water. From the radial position of the raft measured in the experiment, we can therefore calculate its vertical position, and finally deduce the total speed $V$, as illustrated in the inset of Fig. 1(b). The curves have to be read from right to left, with an increase of the speed as the raft gets closer to the cylinder, until a maximum from which it decreases shortly. As demonstrated in the inset, it is crucial to take into account the vertical speed of the raft in order to avoid an error that can go up to $50 \%$.

Particles at the edge of the raft are subjected to three forces: the force exerted by the raft itself $F_{\text {raft }}$, which we wish to determine, the force exerted by the cylinder $F_{\text {cyl }}$, through the slope of the interface, and finally the hydrodynamic forces resulting from the motion of the raft $F_{\text {drag }}$, which we deduce from the experiment. Experimentally, we observe that the erosion mostly takes place at the rear of the raft. Indeed, from a purely geometrical point of view, a particle strictly at the rear of the raft is free to detach very easily, whereas a particle on either side of the raft is blocked by the presence of neighboring particles and is more likely to slide and rearrange along the raft than to detach. As a consequence, we will write the equilibrium for the farthest particle from the cylinder [see the particle encircled in red in Fig. 1(a)], for which all forces are aligned.

First, following the linear approach described in Refs. [17,18], and assuming small deformations and small particles in regard to the capillary length $\ell_{c}=\sqrt{\gamma /\left[\left(\rho_{w}-\rho_{o}\right) g\right]}$ (where $g$ is the acceleration of gravity), we can roughly estimate $F_{\text {cyl }}$ as the product of the weight of the particle with the slope of the interface:

$$
F_{\mathrm{cyl}}=\frac{4}{3} \pi R_{\mathrm{part}}^{3}\left(\rho_{s}-\frac{\rho_{w}+\rho_{o}}{2}\right) g \frac{d h}{d r}\left(r=L+R_{\mathrm{raft}}\right),
$$

where $h$ stands for the depth of the interface perturbed by the cylinder but without the presence of the raft.

The hydrodynamic forces $F_{\text {drag }}$ need a careful treatment. By considering the flow around the red particle in Fig. 1(a), we can calculate a Reynolds number based on the particle radius $\operatorname{Re}_{\text {part }}=R_{\text {part }} V_{\text {raft }} / \nu \ll 1$ for all our experiment, and a second one based on the raft radius $\operatorname{Re}_{\text {raft }}=$ $R_{\text {raft }} V_{\text {raft }} / \nu \approx 1$ when the speed is maximum (for instance, $\mathrm{Re}_{\text {raft }} \approx 3$ for the maximum speed of Fig. 1). We thus typically expect the flow field to be completely laminar and the Stokes law to be valid.

This leads to a Stokes drag that can be expressed as follows:

$$
F_{\text {drag }}=6 \pi \mu k R_{\text {part }} V_{\text {raft }}
$$

with $\mu$ the dynamic viscosity of the oil phase, and $k$ a coefficient that accounts both for the fact that the particle moves along an interface, and as a consequence is immersed in two phases, and for the screening of the drag experienced by the bead because of the rest of the granular raft. We assume the coefficient $k$ is of the order of 0.5 . In reality, the combined effects of the screening and the presence of two phases will lead to a lower coefficient, but the screening being complex to evaluate, we choose to keep $k=0.5$ for all our experiments. In Ref. [10], where a similar approach 


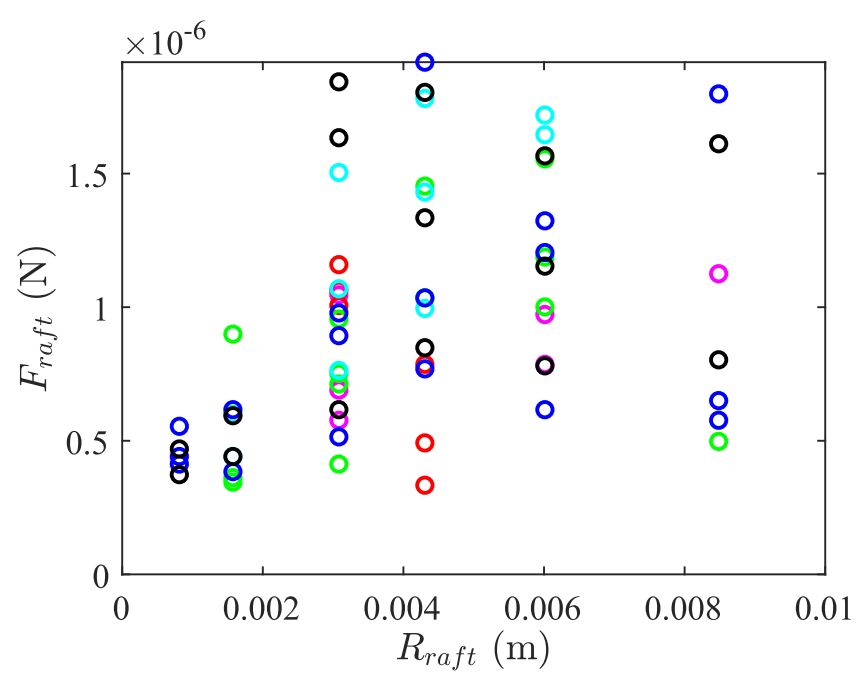

FIG. 2. Critical force needed to erode a granular raft and detach a particle, as a function of the radius of the raft. The force is calculated from experimental measurements of the raft speed at the exact moment of the first detachment of a bead, as defined in Eq. (5), during an experiment such as the one represented in Fig. 1. The colors stand for the depth of the cylinder $\left(R_{\mathrm{cyl}}=15 \mathrm{~mm}\right)$ in the corresponding experiment (purple: $H_{\text {cyl }}=6 \mathrm{~mm}$, red: $H_{\text {cyl }}=7 \mathrm{~mm}$, green: $H_{\text {cyl }}=8 \mathrm{~mm}$, light blue: $H_{\text {cyl }}=9 \mathrm{~mm}$, dark blue: $H_{\text {cyl }}=10 \mathrm{~mm}$, black: $\left.H_{\mathrm{cyl}}=11 \mathrm{~mm}\right)$.

is developed, the coefficient $k$ is taken as a fitting coefficient and is found to remain in the interval $[0.2,0.3]$ for the oil-water interface. Moreover, as illustrated by Fig. 1, the raft does not move at a constant speed. The transient nature of its motion could influence the erosion. Yet, by measuring the acceleration of the raft, we can estimate the amplitude of the inertial forces, which appear to be more than one order of magnitude lower than the Stokes drag, and as a consequence can be neglected. One last assumption made here is that the velocity remains the same for every particle forming the raft. However, since the local curvature of the interface increases as the raft gets closer to the cylinder, we could expect the front of the raft to go faster than its rear, leading to an additional tangential stress that could stretch the raft. To address this issue, we measure the length of a raft $L_{\text {raft }}$ as a function of $L, L_{\mathrm{raft}}$ being defined as the distance between the particle closest to the cylinder and the farthest one. Here again, we solve numerically Eq. (1) to deduce from top views of the raft its arclength along the curved interface. We measured $L_{\text {raft }}$ for several sizes of rafts and several depths of the cylinder (for situations where erosion was observed). For low deflections of the interface, $L_{\text {raft }}$ is globally constant. For the highest $H_{\text {cyl }}, L_{\text {raft }}$ changes by no more than the size of one particle, even for the longest raft. As a consequence, considering the axisymmetric rafts studied in the present paper we believe we can neglect the differential velocity across the area of the raft.

Keeping in mind that the drag force may be slightly overestimated, we write the balance of forces at the exact moment of detachment of the particle $t_{\text {crit }}$, when the three forces are at equilibrium:

$$
\begin{gathered}
F_{\text {raft }}=F_{\text {drag }}\left(t=t_{\text {crit }}\right)-F_{\text {cyl }}\left(t=t_{\text {crit }}\right), \\
F_{\text {raft }} \approx F_{\text {drag }}\left(t=t_{\text {crit }}\right),
\end{gathered}
$$

where we simplify Eq. (4) into (5) by using the fact that experimentally, we always have $F_{\text {drag }}(t=$ $\left.t_{\text {crit }}\right) \gg F_{\text {cyl }}\left(t=t_{\text {crit }}\right)$. For every experiment where a detachment is observed, we calculate $F_{\text {drag }}(t=$ $\left.t_{\text {crit }}\right)$, and plot it as a function of $R_{\text {raft }}$, as represented in Fig. 2 . We get a lot of points for any given $x$-axis coordinate, since we explore a large range of values for $H_{\text {cyl }}$, and because the same experiment is reproduced several times for each given depth. For each set of parameters $\left(R_{\text {raft }}, H_{\text {cyl }}\right)$ the critical 


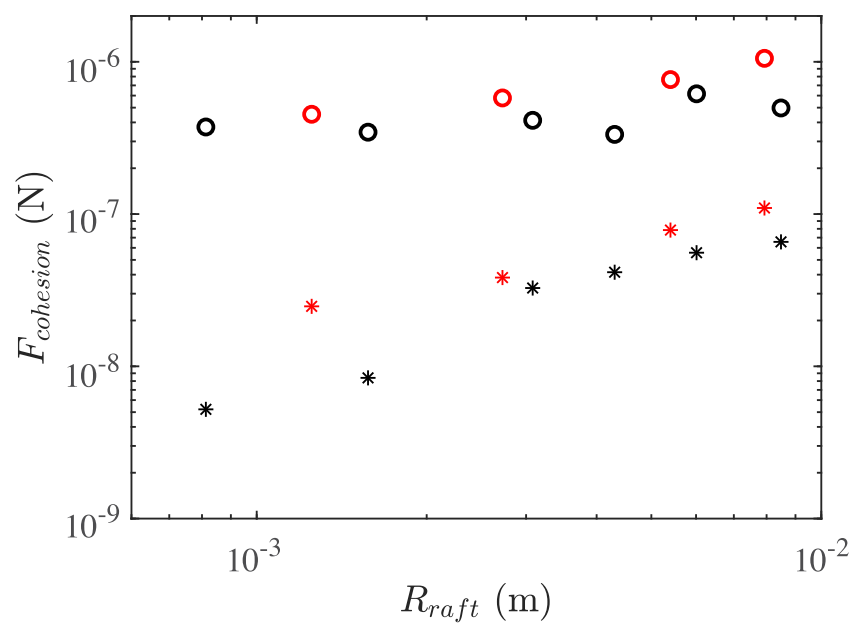

FIG. 3. Minimal cohesion force of a raft (circles), compared to the theoretical capillary force a raft should exert on a particle at its edge, as defined in Eqs. (2) and (6) (stars), as a function of the radius of the raft, for two different types of ceramic beads (black: $\rho_{s}=3800 \mathrm{~kg} \mathrm{~m}^{-3}, R_{\text {part }}=0.125 \mathrm{~mm}$, red: $\rho_{s}=6000 \mathrm{~kg} \mathrm{~m}^{-3}$, $R_{\text {part }}=0.1 \mathrm{~mm}$ ). The circles are obtained by taking for each raft radius the minimal value of $F_{\text {crit }}$ in Fig. 2 for the black circles (and of a similar set of data for the other type of particles).

speed at which erosion occurs varies significantly from one experiment to the other, as emphasized by the great dispersion of the data of a given color at a fixed $x$-axis coordinate. Moreover, the speed a raft increases with its size, as demonstrated in previous work [19]. As a consequence, a bead belonging to the smallest raft will never experience a drag force as high as a particle inside a larger raft, leading to a smaller scattering of the data.

Figure 2 gives information on the cohesion force of a raft $\left(F_{\text {raft }} \approx 0.5-2 \times 10^{-6} \mathrm{~N}\right)$, since it represents the force needed to extract a particle from the raft. However no clear trend can be extracted from this data, especially knowing that for each $x$-axis coordinate, erosion was sometimes not observed, meaning the raft speed was not high enough to generate a drag force greater than the cohesion force. In other words, these experiments correspond to data points in Fig. 2 which may go beyond the limit of the $y$ axis and that we cannot observe with the experimental setup described here. The arrangements of the particles inside each raft may differ greatly from one experiment to the other and thus lead to strong differences in the global cohesion force. Because of this limitation, it would make no sense to average the critical force for each raft radius. But by taking the minimum value for each $x$-axis coordinate, we deduce a lower boundary for the cohesion force of a granular raft as a function of its radius, as represented in Fig. 3 for two types of particles, with a slight increase of $F_{\text {cohesion }}$ with $R_{\text {raft }}$.

To account for these experimental points, we can estimate the capillary force a raft exerts on a particle at its edge. This force can be estimated similarly to Eq. (2), as the product between the weight of a particle and the slope of the interface at the edge of a raft. This slope can either be measured on side views of the rafts or by solving a linearized version of Eq. (1), leading to the following result [19]:

$$
\frac{d h_{\mathrm{raft}}}{d r}\left(r=R_{\mathrm{raft}}\right)=\frac{h_{\mathrm{raft}} / \ell_{c}}{K_{0}\left(R_{\mathrm{raft}} / \ell_{c}\right)} K_{1}\left(\frac{R_{\mathrm{raft}}}{\ell_{c}}\right),
$$

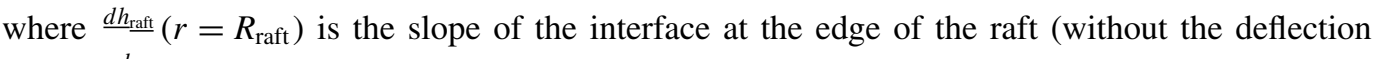
$d r$ caused by the cylinder), $h_{\text {raft }}$ the depth of the edge of the raft, and $K_{i}$ the modified Bessel function of

the second kind of order $i$. The slope remaining rather small for the sizes explored

experimentally, 


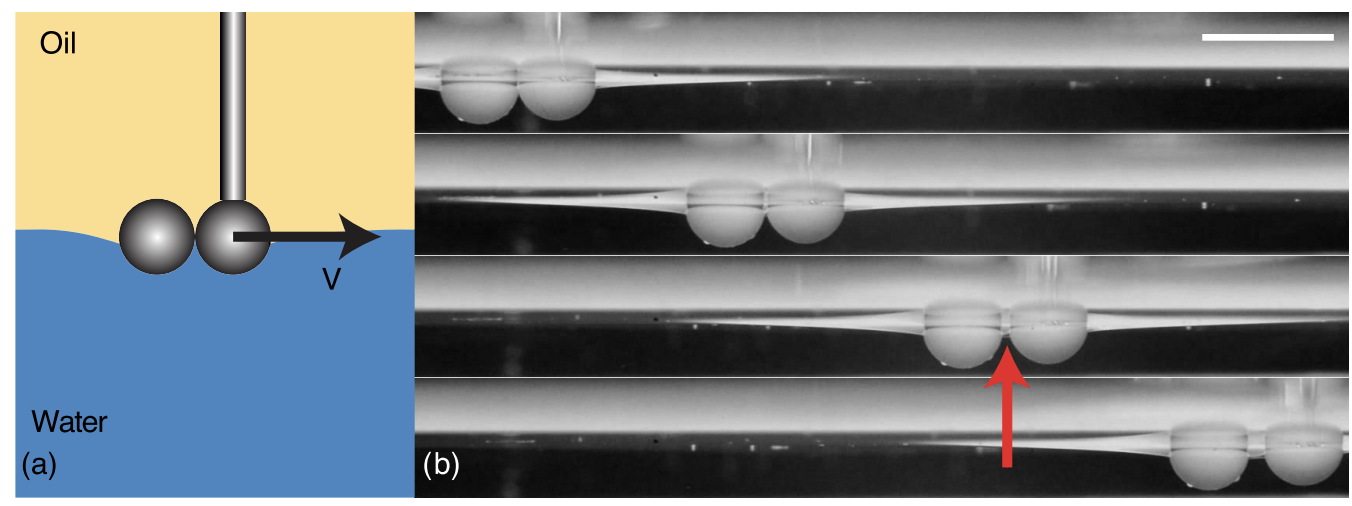

FIG. 4. Force of cohesion between two particles at an interface: experimental setup and visualization of a typical experiment. (a) Schematic representation of the experimental configuration. A metallic wire is glued on a bead which is then placed at the interface and attracts another particle. The two aggregated particles are then translated at a constant speed $V$. (b) Time lapse of the translation of a couple of plastic particles $\left(\rho_{s}=\right.$ $1420 \mathrm{~kg} \mathrm{~m}^{-3}, R_{\text {part }}=1.5 \mathrm{~mm}$ ) at an oil-water interface, at the critical speed of detachment $V_{\text {crit }}=6 \mathrm{~mm} / \mathrm{s}$. The red arrow indicates the separation between the two particles. The movie is taken from the side. Time between two images: $1.6 \mathrm{~s}$. Scale bar: $5 \mathrm{~mm}$ (see Supplemental Material Movie 2 [16]).

the theoretical slope predicted by Eq. (6) and its experimental value differ by less than $10 \%$. The corresponding force is represented by red and black stars in Fig. 3.

We find a strong disagreement between the experiments and the theoretical results for the measured cohesion force. As pointed out previously, the force is probably overestimated but the screening is not sufficient to account for the two orders of magnitude of difference between the theory and the experiment for the smallest raft. Indeed, the screening derived in Ref. [10] would lower our data only by a factor 2 or 3 , which would be far from sufficient to conciliate the experiment with the theory. Unfortunately, our experimental setup does not allow us either to go further in the understanding of what seems to be an unexpectedly high cohesion or to undoubtedly exclude the possibility of a screening higher than expected. Moreover, friction due to the solid-solid contact between the spheres as well as jamming phenomena preventing particles from moving can be observed and should be included to completely describe the cohesion of a raft.

\section{COHESION OF A PAIR OF PARTICLES AT AN INTERFACE}

To circumvent these issues we now focus on the characterization of the cohesion between two spheres at an oil-water interface. In that model situation, the capillary interaction can be described more finely, the screening will be less important a priori, and no jamming due to the specific arrangement of the beads is possible. Moreover, for two particles, the linear approximation is completely valid, giving more credibility to the capillary force calculated. With the current experimental device, the speed a two-bead aggregate can reach during its interaction with a cylinder is too low to separate the particles. In order to play directly on the drag experienced instead of applying an external force field to change indirectly the speed, the experimental setup of Fig. 4(a) is developed.

A metallic wire with a diameter of $0.3 \mathrm{~mm}$ is glued on a bead and connected to a vertical manual translation stage, itself fixed on a horizontal Thorlabs linear translation stage mounted with a stepped motor, that can reach a speed $V=30 \mathrm{~mm} / \mathrm{s}$ along a travel range of $150 \mathrm{~mm}$. The particle is then placed at an oil-water interface, at the depth of a freely floating pair of particles (which is determined experimentally), and another identical bead is added not far and aggregates with the wire-connected particle. Due to this procedure, the pair of particles has the same vertical position it would have had 


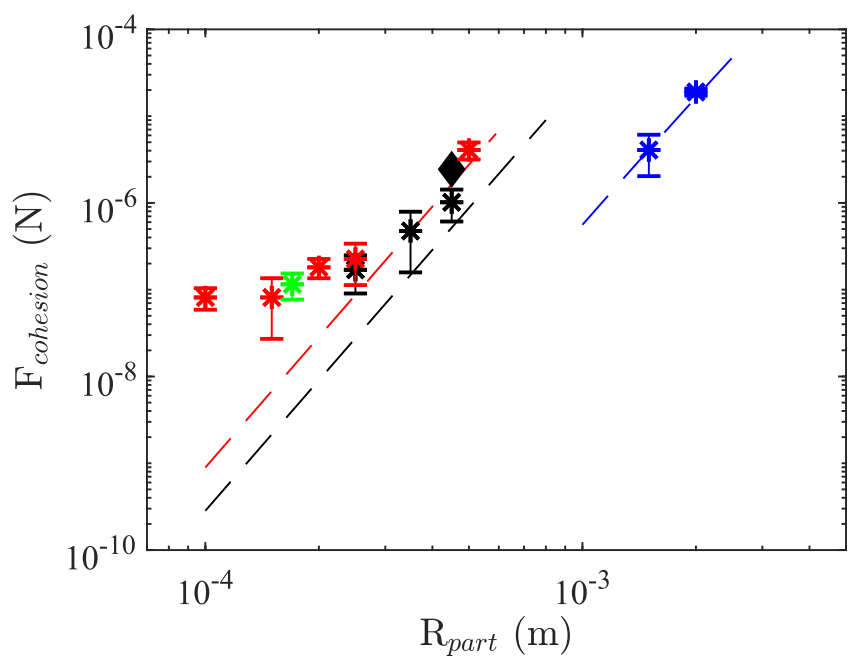

FIG. 5. Experimental cohesion force of a pair of particles calculated due to Eq. (3) (colored stars), compared with the theoretical capillary force between two beads (dotted lines), as defined in Eq. (7), as a function of the radius of the beads, for different types of particles (black: ceramic beads $\rho_{s}=3800 \mathrm{~kg} \mathrm{~m}^{-3}$, red: high-density ceramic beads $\rho_{s}=6000 \mathrm{~kg} \mathrm{~m}^{-3}$, blue: plastic beads $\rho_{s}=1420 \mathrm{~kg} \mathrm{~m}^{-3}$, green: glass beads $\rho_{s}=2500 \mathrm{~kg} \mathrm{~m}^{-3}$ ). The black diamond at $R_{\text {part }}=0.45 \mathrm{~mm}$ corresponds to an experiment done with a larger wire (diameter of $1.1 \mathrm{~mm}$ ).

without the presence of the wire. The pair of beads is then moved horizontally at a given constant speed $V$. If no detachment is observed, the speed is increased gradually from one experiment to the next until the two beads separate. The horizontality of the translation stage being fundamental to keep the particles at the same level along their motion, a highly sensitive spirit level with a precision of $0.1 \mathrm{mrad}$ is used. No clear influence of the acceleration ramp was noted: for any given experiment, the detachment velocity is the same whatever the acceleration needed to reach this velocity is, in the range $[015] \mathrm{mm} / \mathrm{s}^{2}$.

Several types of particles are used in this experiment: plastic beads $\left(\rho_{s}=1420 \mathrm{~kg} \mathrm{~m}^{-3}, R_{\text {part }} \in\right.$ $[2.5,2,1.5] \mathrm{mm})$, ceramic beads $\left(\rho_{s}=3800 \mathrm{~kg} \mathrm{~m}^{-3}, R_{\text {part }} \in[0.45,0.35,0.25] \mathrm{mm}\right)$, high-density ceramic beads $\left(\rho_{s}=6000 \mathrm{~kg} \mathrm{~m}^{-3}, R_{\text {part }} \in[0.5,0.25,0.2,0.15,0.1] \mathrm{mm}\right)$, and finally glass beads $\left(\rho_{s}=2500 \mathrm{~kg} \mathrm{~m}^{-3}, R_{\text {part }}=0.17 \mathrm{~mm}\right)$. A typical experiment is shown in Fig. 4(b), where two plastic beads are moved horizontally at the critical detachment speed. The freely floating particle slowly detaches from the wire-connected one, a behavior which was not observed for a lower speed. No detachment was observed for the biggest plastic bead, for which the experimental device could not reach high enough velocities.

The drag force experienced by the detaching particle is estimated by Eq. (3), exactly as before, with the same coefficient $k=0.5$. At detachment, since only the drag and the attractive cohesive forces are present, this estimation directly gives us the cohesion force between the two beads. The results are displayed in Fig. 5. The error bars of our data are determined by reproducing several times the experiment first in the same conditions, then with slightly different depths of the beads (the depth was changed by no more than 10\%), in order to determine lower and upper boundaries of this critical speed.

These experimental points need to be compared to the theoretical prediction of the capillary force between two particles. In the limit of small deformations, small spherical particles, and isotropy of the meniscus around a sphere, the capillary force between two identical spheres in contact at an 
interface can be expressed as follows [18,20]:

$$
F_{\text {cap }}=2 \pi \gamma R_{\text {part }} \mathrm{Bo}^{5 / 2} \Sigma^{2} K_{1}\left(\frac{2 R_{\text {part }}}{\ell_{c}}\right)
$$

with $\mathrm{Bo}=\left(R_{\text {part }} / \ell_{c}\right)^{2}$ the Bond number, $\Sigma=\frac{2 D-1}{3}-\frac{1}{2} \cos \theta+\frac{1}{6} \cos ^{3} \theta, \theta$ the oil-water-particle contact angle, and $D=\frac{\rho_{s}-\rho_{o}}{\rho_{w}-\rho_{o}}$. This theoretical cohesion force is represented in dotted lines in Fig. 5. For the biggest particles, this linear theory perfectly accounts for our results, and the experimental cohesion force strictly corresponds to the capillary attraction between the two beads. However, as the radius of the beads decreases, the disagreement greatly increases, up to two orders of magnitude for the smallest beads used. This result is consistent with the previous experiment with a granular raft, where the same small particles were used (red points of Fig. 3), and a similar disagreement was found between the classic linear capillary theory and the experimental cohesion force measured. However, in the situation presented here, the assumptions needed to derive Eq. (7) are even more valid. Furthermore, contrary to the erosion experiment, no jamming between particles is possible, and the screening of the drag created by the other particle cannot account for two orders of magnitude.

The last source of error in the interpretation of the experiment could come from the presence of the metallic wire, which could have an influence on the flow. A priori the larger the wire is with respect to the particle, the more it will affect the drag experienced. In order to probe its influence, we used two diameters of wire for one of the experiment $\left(\rho_{s}=3800 \mathrm{~kg} \mathrm{~m}^{-3}, R_{\text {part }}=0.45 \mathrm{~mm}\right)$ : the usual wire with a diameter of $0.3 \mathrm{~mm}$, and a larger one with a diameter of $1.1 \mathrm{~mm}$, whose size is comparable to the particle. There is only a factor 2 of difference between the two cohesion forces deduced from these two experiments (black star and black diamond at $R_{\text {part }}=0.45 \mathrm{~mm}$ ). This tends to prove that even though the wire does affect the detachment, it cannot explain the two orders of magnitude found for the smallest bead.

This result indicates that a more fundamental explanation has to be found. The answer could lie within the assumptions needed for the calculation of Eq. (7), and especially under the hypothesis of isotropy, which is not clear for two particles in contact.

In 2017 Cooray et al. [21] calculated the capillary forces for a pair of particles without assuming axisymmetry around a particle, precisely by taking into account the tilting of the contact line around each sphere. By doing so, two components of the force arise:

(1) A hydrostatic force $F_{\text {hyd }}$, due to the difference of depth of the two phases around the sphere, when integrating the pressure

(2) A surface tension force $F_{\mathrm{st}}$, resulting from the integration of surface tension along the nonplanar contact line.

Both forces have vertical and horizontal components, but here we will focus only on the horizontal ones. Following the calculations detailed in Refs. [21,22], we end up with expressions for $F_{\text {hyd }}^{(x)}$ and $F_{\text {st }}^{(x)}$ that depend only on the contact angle $\theta$, the position of the contact line for an isolated sphere $\varphi_{s}$, the radius of the particle $R_{\text {part }}$, and the fluid parameters (for the exact expressions, see Supplemental Material [16]), as defined in Fig. 6(a). The resultant horizontal force is

$$
F^{(x)}=F_{\text {hyd }}^{(x)}+F_{\text {st }}^{(x)} \text {. }
$$

To calculate $F^{(x)}$, we need the values of $\theta, \varphi_{s}$, and $R_{\text {part }} . \theta$ is measured on side views of a floating particle, while $\varphi_{s}$ can be either calculated or measured, as represented in Fig. 6(b). The calculation comes from a vertical balance of the three forces acting on a particle: the weight, the buoyancy, and the surface tension force. Without any assumptions, this leads to [21]

$$
\begin{aligned}
\frac{4}{3} D= & \frac{2}{3}-\cos \varphi_{s}+\frac{1}{3} \cos ^{3} \varphi_{s}-\frac{\xi_{s}}{\mathrm{Bo}^{1 / 2} \ell_{c}} \sin ^{2} \varphi_{s} \\
& -\frac{2}{\mathrm{Bo}} \sin \varphi_{s} \sin \left(\varphi_{s}+\theta\right)
\end{aligned}
$$



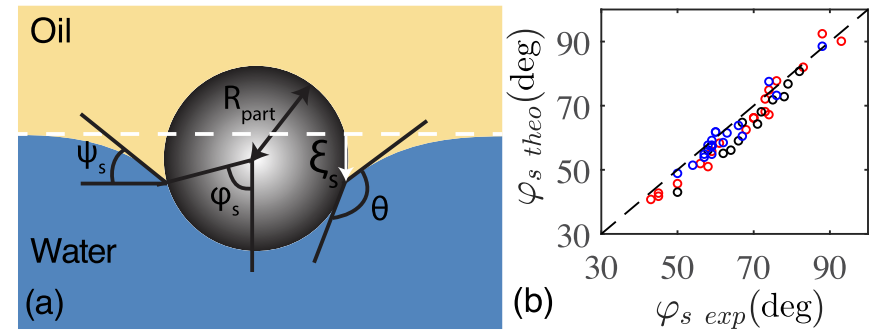

FIG. 6. (a) Schematic representation of a particle at an oil-water interface, along with some important parameters that we need to take into account for the calculation of the capillary forces. (b) Theoretical depth angle $\varphi_{s}$ theo as a function of the experimental one $\varphi_{s \text { exp }}$, for different types of particles (black: ceramic beads $\rho_{s}=3800 \mathrm{~kg} \mathrm{~m}^{-3}$, red: high-density ceramic beads $\rho_{s}=6000 \mathrm{~kg} \mathrm{~m}^{-3}$, blue: plastic beads $\rho_{s}=1420 \mathrm{~kg} \mathrm{~m}^{-3}$ ). The dotted line of slope one is only a guideline for the eye.

with $\xi_{s}$ the depth of the contact line, as defined in Fig. 6(a). In the limit of small deformations, the equation of the interface we need to solve in order to deduce $\xi_{s}$ is the following:

$$
\begin{aligned}
& \nabla^{2} h=\frac{h}{\ell_{c}^{2}} \\
& h^{\prime}\left(r=R \sin \varphi_{s}\right)=\tan \left(\varphi_{s}+\theta\right) \\
& h(+\infty)=0 .
\end{aligned}
$$

Applying the result in $r=R \sin \varphi_{s}$, we end up with Eq. (11):

$$
\xi_{s}=-\tan \left(\varphi_{s}+\theta\right) \ell_{c} \frac{K_{0}\left(R \sin \varphi_{s} / \ell_{c}\right)}{K_{1}\left(R \sin \varphi_{s} / \ell_{c}\right)}
$$

Equation (9) can then be solved numerically, and the value of $\varphi_{s}$ can then be deduced for any given pair of parameters $\left(R_{\mathrm{part}}, \theta\right)$, with a good agreement with the experiment as demonstrated by Fig. 6(b). For all our measurements, the mean difference between the experimental value of $\varphi_{s}$ and the theoretical one is $\Delta \varphi_{s}=\left\langle\varphi_{s \exp }-\varphi_{s}\right.$ theo $\rangle \approx 3^{\circ}$.

From there, the value of $F^{(x)}$ can be computed only as a function of $\left(R_{\text {part }}, \theta\right)$ and compared to

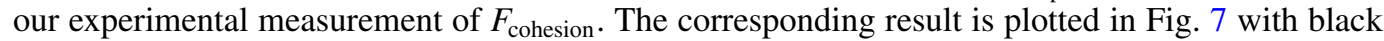
squares, along with the previous experimental result with colored stars, and the first theoretical model of Eq. (7) with a plain line. The rescaling of the $x$ axis comes from the asymptotic analysis of Eq. (7) for small radii [see Eq. (12)], which indicates that $\mathrm{Bo}^{5 / 2} \Sigma^{2}$ is the good rescaling parameter:

$$
F_{\text {cap }} \underset{R \rightarrow 0}{\sim} \gamma \ell_{c} \mathrm{Bo}^{5 / 2} \Sigma^{2} .
$$

Because there is a strong hysteresis of the contact angle (up to $40^{\circ}$ between its minimal and maximal value), we measure $\theta$ with different beads for each radius, and calculate as a consequence several cohesion forces for each radius, depending on $\theta$. Yet the values obtained are almost not affected by this hysteresis. The force obtained differs only slightly from the simple calculation of Eq. (7) (not more than 10\% of difference, which is consistent with the results reported in Ref. [21]). As a consequence, this approach does not explain our experimental result.

As pointed out before, $\varphi_{s}$ exp differs from its theoretical value by $\Delta \varphi_{s} \approx 3^{\circ}$. We can therefore shift all depth angles by a constant value $\Delta \varphi_{s}$ and calculate $F^{(x)}\left(R_{\text {part }}, \theta_{\text {mean }}, \varphi_{s}\right.$ theo $\left.+\Delta \varphi_{s}\right)$. The corresponding result is plotted in dotted lines in Fig. 7 (we also use one single value of $\theta_{\text {mean }}$ for each material).

Surprisingly, considering the small value of $\Delta \varphi_{s}$, both the general trend and the order of magnitude are now consistent with the experiments: for small radii, we recover an enhanced 


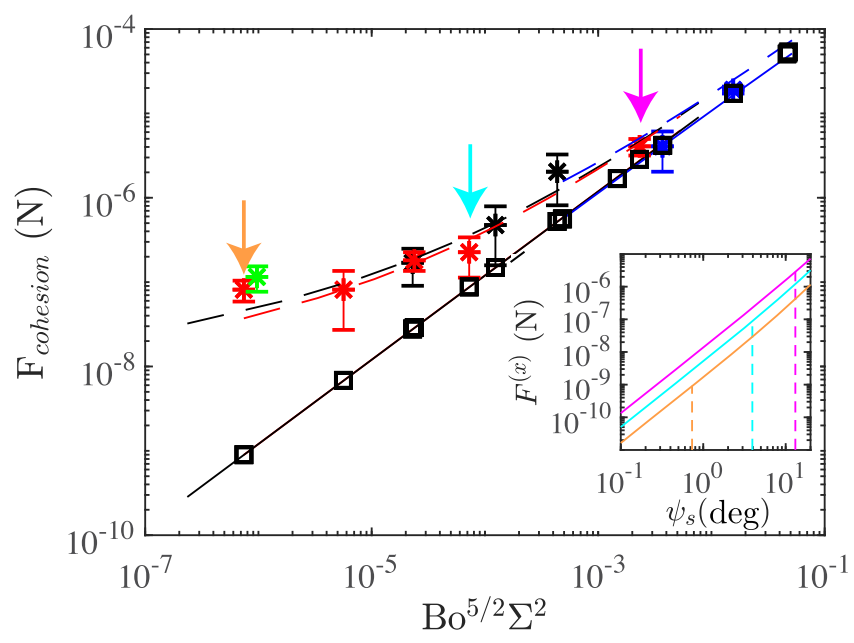

FIG. 7. Experimental cohesion force of a pair of particles calculated due to Eq. (3) (colored stars), compared with three theoretical predictions: the classical capillary force between two beads $F_{\text {cap }}$ (plain line), as defined in Eq. (7), the full solving of the force $F^{(x)}$ from Eq. (8), taking into account the tilting of the contact line, with the theoretical depth angle $\varphi_{s}$ theo given by Eq. (9) (black squares), and finally with dotted lines the same horizontal force $F^{(x)}$ but with a depth angle of $\varphi_{s}$ theo $+3^{\circ}$. The data are plotted as a function of the dimensionless number $\mathrm{Bo}^{5 / 2} \Sigma^{2}$, as defined in Eq. (7), for different types of particles (black: ceramic beads $\rho_{s}=3800 \mathrm{~kg} \mathrm{~m}^{-3}$, red: high-density ceramic beads $\rho_{s}=6000 \mathrm{~kg} \mathrm{~m}^{-3}$, blue: plastic beads $\rho_{s}=1420 \mathrm{~kg} \mathrm{~m}^{-3}$, green: glass beads $\rho_{s}=2500 \mathrm{~kg} \mathrm{~m}^{-3}$ ). The colored arrows show which three points have been selected for the inset. Inset: Theoretical horizontal force between two ceramic beads, as a function of the slope of the interface $\psi_{s}$, for three different radii (magenta: $R_{\text {part }}=0.5 \mathrm{~mm}$, light blue $R_{\text {part }}=0.25 \mathrm{~mm}$, orange $R_{\text {part }}=0.1 \mathrm{~mm}$ ). The dotted lines indicate the theoretical slopes $\psi_{s}$ theo for each radius (the corresponding theoretical force can be read in ordinate).

capillary force, whereas when the radius of the particle increases, both calculations lead to the same result. This suggests that the small variations of $\varphi_{s}$ have a strong effect on $F^{(x)}$ for small particles, an interpretation that needs some backup.

Up to now, we have considered $F^{(x)}$ for fixed values of $\varphi_{s}$. As represented in the inset of Fig. 7, we investigate how $F^{(x)}$ varies with the angle $\psi_{s}=\varphi_{s}+\theta-\pi$, with $\psi_{s}$ defined in Fig. 6(a) (simple geometrical considerations lead to this relation). The three curves correspond to three different radii of particles, and the dotted lines indicate the theoretical angles $\psi_{s}$ theo $=\varphi_{s}$ theo $+\theta-\pi$ for each radius. As illustrated, $F^{(x)} \propto \psi_{s}^{2}$ for small angles. As a consequence, a variation of $\Delta \varphi_{s}$ in the $x$ axis for the bigger radius in pink would result in only a factor two or three for the force, while the very same variation on the $x$ axis for the smallest particle in green would result in more than one order of magnitude in ordinate. Thus, as $\psi_{s}$ gets smaller, the tiniest variation of its value has an increasing effect on $F^{(x)}$.

The last remaining question concerns the differences between $\varphi_{s} \exp$ and $\varphi_{s}$ theo. The approximation made in the calculation of $\varphi_{s}$ theo is perfectly valid for the smallest particles considered. Yet it is well known that for small particles, capillary forces between objects are produced via geometrical details such as the object's shape or surface roughness [23,24]. Such undulations of the contact line may also be present for larger particles, leading to an effective depth angle $\varphi_{s}$ exp different from the theoretical one by only a few degrees. However, as demonstrated earlier, these few degrees can lead to very strong variations of the capillary force between two spheres in contact. The tilting of the contact line as the two particles come into contact also plays an essential role in the observed large increase of the capillary forces. It is the combination of these two effects that leads to an enhanced cohesion force for small particles. 
This result for the cohesive force between two beads can be extrapolated to explain qualitatively the cohesion of a whole raft, which is higher than the capillary force estimated theoretically. This unexpectedly high cohesive force thus means these aggregates are far more resistant than expected and can resist far stronger flows.

\section{CONCLUSIONS AND OUTLOOK}

In conclusion, we have investigated the cohesion inside an assembly of large dense particles at an interface and between two aggregated beads at a liquid interface. The erosion a granular raft experiences during its motion occurs for unexpectedly high speeds. This enhanced cohesion appears to result from the precise description of the meniscus around each particle, as demonstrated by the two-bead experiment. For small particles, the attractive force between two beads in contact exceeds by two orders of magnitude the force expected by the classic theory, whereas no significant effect is observed for large particles. The tilting of the contact line as well as small variations in the pinning of the contact line seem to explain this enhanced force.

The small differences between the theoretical depth angle of a freely floating particle and the experimental one requires some further investigation. In particular, the role of roughness, nonspherical shapes, and surface inhomogeneity on the effective depth angle deserves to be investigated further. The present study could also be extended to the capillary forces between smaller particles or to the erosion of particle-covered droplets under flow. A theoretical description of the geometry of the contact line when more than two beads are in contact along a curved interface would help us to understand this phenomenon.

\section{ACKNOWLEDGMENT}

We gratefully thank Christophe Josserand for many insightful conversations at various stages of this work.

[1] H. Hoekstra, J. Vermant, J. Mewis, and G. Fuller, Flow-induced anisotropy and reversible aggregation in two-dimensional suspensions, Langmuir 19, 9134 (2003).

[2] A. Zaccone, M. Soos, M. Lattuada, H. Wu, M. U. Bäbler, and M. Morbidelli, Breakup of dense colloidal aggregates under hydrodynamic stresses, Phys. Rev. E 79, 061401 (2009).

[3] C. Camoin and R. Blanc, Aggregation in a sheared 2D dispersion of spheres with attractive interactions, J. Phys. Lett. 46, 67 (1985).

[4] E. J. Stancik, G. T. Gavranovic, M. J. Widenbrant, A. T. Laschitsch, J. Vermant, and G. G. Fuller, Structure and dynamics of particle monolayers at a liquid-liquid interface subjected to shear flow, Faraday Discuss. 123, 145 (2003).

[5] E. J. Stancik, M. J. Widenbrant, A. T. Laschitsch, J. Vermant, and G. G. Fuller, Structure and dynamics of particle monolayers at a liquid-liquid interface subjected to extensional flow, Langmuir 18, 4372 (2002).

[6] D. Vella, P. Aussillous, and L. Mahadevan, Elasticity of an interfacial particle raft, Europhys. Lett. 68, 212 (2004).

[7] E. Jambon-Puillet, C. Josserand, and S. Protière, Wrinkles, folds, and plasticity in granular rafts, Phys. Rev. Mater. 1, 042601 (2017).

[8] K. Higashitani and K. Iimura, Two-dimensional simulation of the breakup process of aggregates in shear and elongational flows, J. Colloid Interface Sci. 204, 320 (1998).

[9] N. D. Vassileva, D. van den Ende, F. Mugele, and J. Mellema, Restructuring and break-up of twodimensional aggregates in shear flow, Langmuir 22, 4959 (2006).

[10] N. D. Vassileva, D. van den Ende, F. Mugele, and J. Mellema, Fragmentation and erosion of twodimensional aggregates in shear flow, Langmuir 23, 2352 (2007). 
[11] M. Abkarian, S. Protière, J. M. Aristoff, and H. A. Stone, Gravity-induced encapsulation of liquids by destabilization of granular rafts, Nat. Commun. 4, 1895 (2013).

[12] S. Protière, C. Josserand, J. M. Aristoff, H. A. Stone, and M. Abkarian, Sinking a Granular Raft, Phys. Rev. Lett. 118, 108001 (2017).

[13] P. A. Kralchevsky and K. Nagayama, Capillary interactions between particles bound to interfaces, liquid films and biomembranes, Adv. Colloid Interface Sci. 85, 145 (2000).

[14] M. Cavallaro, L. Botto, E. P. Lewandowski, M. Wang, and K. J. Stebe, Curvature-driven capillary migration and assembly of rod-like particles, Proc. Natl. Acad. Sci. U.S.A. 108, 20923 (2011).

[15] I. B. Liu, N. Sharifi-Mood, and K. J. Stebe, Capillary assembly of colloids: Interactions on planar and curved interfaces, Annu. Rev. Condens. Matter Phys. 9, 283 (2018).

[16] See Supplemental Material at http://link.aps.org/supplemental/10.1103/PhysRevFluids.5.044003 for (i) movie where raft erosion is observed, (ii) a movie where two particles separate from one another, and (iii) a calculation of the capillary forces between two beads in contact.

[17] M. Nicolson, The interaction between floating particles, in Mathematical Proceedings of the Cambridge Philosophical Society, Vol. 45 (Cambridge University Press, 1949), pp. 288-295.

[18] D. Vella and L. Mahadevan, The "Cheerios" effect, Am. J. Phys. 73, 817 (2005).

[19] A. Lagarde, C. Josserand, and S. Protiere, The capillary interaction between pairs of granular rafts, Soft Matter 15, 5695 (2019).

[20] A. Dani, G. Keiser, M. Yeganeh, and C. Maldarelli, Hydrodynamics of particles at an oil-water interface, Langmuir 31, 13290 (2015).

[21] H. Cooray, P. Cicuta, and D. Vella, Floating and sinking of a pair of spheres at a liquid-fluid interface, Langmuir 33, 1427 (2017).

[22] P. L. H. M. Cooray, The capillary interaction between objects at liquid interfaces, Ph.D. thesis, University of Cambridge, 2014.

[23] K. D. Danov, P. A. Kralchevsky, B. N. Naydenov, and G. Brenn, Interactions between particles with an undulated contact line at a fluid interface: Capillary multipoles of arbitrary order, J. Colloid Interface Sci. 287, 121 (2005).

[24] L. Botto, E. P. Lewandowski, M. Cavallaro, and K. J. Stebe, Capillary interactions between anisotropic particles, Soft Matter 8, 9957 (2012). 